ORIGINAL

\title{
Effects of FM system fitted into the normal hearing ear or cartilage conduction hearing aid fitted into the affected ear on speech-in-noise recognition in Japanese children with unilateral congenital aural atresia
}

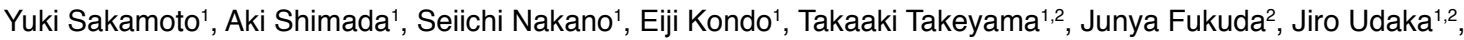 \\ Hidehiko Okamoto ${ }^{3}$, and Noriaki Takeda ${ }^{1}$ \\ ${ }^{1}$ Department of Otolaryngology, Tokushima University Graduate School of Biomedical Sciences, Tokushima, Japan, ${ }^{2}$ Udaka ENT Clinic, \\ Tokushima, Japan, ${ }^{3}$ Department of Physiology, International University of Health and Welfare School of Medicine, Narita, Japan
}

\begin{abstract}
The effects of FM system fitted into the normal hearing ear (NHE) or a cartilage conduction hearing aid (CCHA) fitted into the affected ear (AE) on the speech recognition ability in noise were examined in children with unilateral congenital aural atresia (UCAA). In children with bilateral normal hearing (BNH), speech recognition score (SRS) was significantly decreased in the noisy environment of -5 dB signal-to-noise ratio (SNR), compared with those in quiet. In children with UCAA, SRS was significantly decreased in noisy environments of 0 and -5 dB SNR, compared with those in quiet. In noisy environments of 0 and -5 dB SNR, SRS in children with UCAA was significantly decreased, compared those in children with BNH. In the noisy environment of $-5 \mathrm{~dB}$ SNR, SRS in UCAA children aided by FM system fitted into NHE was significantly better than those in unaided children in the same group. In the noisy environment of $0 \mathrm{~dB}$ SNR, SRS in UCAA children aided by CCHA into AE tended to be higher than those in unaided children in the same group. FM system and CCHA can be recommended as an audiological management for the improvement of speech recognition in children with UCHL in classrooms. J. Med. Invest. $67: 134-138$, February, 2020
\end{abstract}

Keywords : children with unilateral conductive hearing loss, congenital aural atresia, speech recognition, FM system, cartilage conduction hearing aid

\section{INTRODUCTION}

Unilateral congenital sensorineural hearing loss can be identified by universal newborn hearing screenings shortly after birth. In contrast, unilateral conductive hearing loss secondary to congenital aural atresia must be identified on the day of birth. Until recently, it was believed that unilateral hearing loss had little impact on a child's speech and language development. However, it was recently reported that children with unilateral sensorineural hearing loss are at risk of academic failure $(1,2)$.

Children with unilateral congenital aural atresia have moderate or moderate-severe conductive hearing loss on the affected side. Children with unilateral conductive hearing loss secondary to congenital aural atresia are also suggested to be at greater risk of speech and learning difficulties than previously appreciated; their risk level is similar to children with unilateral sensorineural hearing loss (3). It has been reported that almost two-thirds of children with unilateral congenital aural atresia need some sort of resource assistance, including the use of the FM system in the classroom (4). Because congenital aural atresia is always accompanied by a microtia, conventional hearing aids cannot be used. Bone conduction hearing aids are an alternate device for children with congenital aural atresia. However, its high contact pressure on the mastoid skin usually cause local pain and discomfort. BAHA is also effective, but surgery is

Received for publication December 1, 2019 ; accepted January 9, 2020.

Address correspondence and reprint requests to Noriaki Takeda M.D., Ph.D., Department of Otolaryngology, University of Tokushima School of Medicine, 3-18-15 Kuramoto, Tokushima 770-8503, Japan and Fax : +81-88-633-7170. needed. Recently, cartilage conductive hearing aids have been developed in Japan. It is reported that cartilage conductive hearing aids, which are attached to the cartilage of microtia with double-stick tape are beneficial for aural atresia ear $(5,6)$.

In the present study, we first examined the speech recognition ability of elementary school- and preschool-aged Japanese children with unilateral conductive hearing loss secondary to congenital aural atresia in noisy environments and compared it with that of age-matched children with bilateral normal hearing. We then examined the effects of the FM system fitted into the normal hearing ear of elementary school-aged children with unilateral congenital aural atresia on the speech recognition ability in noisy environments similar to the listening condition of elementary school classrooms in Japan. Finally, we examined the effects of a cartilage conduction hearing aid fitted into the affected ear of children with unilateral congenital aural atresia on the speech recognition ability in the noisy environments.

\section{PATIENTS AND METHODS}

Patients

Eight children with unilateral congenital aural atresia (6 males and 2 females, mean age : $6.2 \pm 1.1$ years) and twelve agematched children with bilateral normal hearing (5 males and 7 females, mean age : $8.2 \pm 1.1$ years) were enrolled in the present study.

The children with unilateral congenital aural atresia showed unilateral moderate conductive hearing loss (mean hearing level : $69.4 \pm 3.3 \mathrm{~dB}$, right : 7 and left : 1 , bony atresia : 7, fibrous atresia : 1). Unilateral atresia in the group was accompanied by a microtia in all cases. 
All children received the speech recognition test in both quiet and noisy environments. Then, because the FM system can be used in the classroom setting, four elementary school-aged children with unilateral congenital aural atresia (3 males and $1 \mathrm{fe}-$ male, mean age : $7.1 \pm 0.8$ years) received the speech recognition test with or without an FM receiver fitted to the normal hearing ear in both quiet and noisy environments. Finally, five children with unilateral congenital aural atresia (4 males and 1 female, mean age : $7.0 \pm 2.1$ years) received the speech recognition test with or without a cartilage conduction hearing aid fitted into the affected ear in both quiet and noisy environments. Characteristics and indications of the FM system and cartilage conduction hearing aids were explained for children and their parents using references (7) and (8), respectively.

The present study was approved by the Committee for Medical Ethics of Tokushima University Hospital, and written informed consent was obtained from a parent of each child prior to the study.

\section{Speech recognition test in noisy environments}

The speech recognition test in a sound field was performed in the audiometric testing room of our otolaryngology clinic and the speech recognition score were measured according to the guidelines for the evaluation of hearing aid fitting (2010) published by the Japan Audiological Society (9). Japanese monosyllables from the 57-S word list made by the Japan Audiological Society were used as the speech material (10). Each monosyllable was presented at $50 \mathrm{~dB}$ time-averaged sound pressure level (SPL) from a loudspeaker placed in front of a subject sitting at a distance of $80 \mathrm{~cm}$ in a quiet environment. The same test was then presented in noisy environments of $0 \mathrm{~dB}$ and $-5 \mathrm{~dB}$ signal-to-noise ratio (SNR), in which white noise (WN) was presented at 50 and 55 dB SPL from another loudspeaker placed behind the subject at a distance of $80 \mathrm{~cm}$ (Fig. 1A). The test condition of speech recognition test in noise, which was published in the guideline for the evaluation of hearing and fitting (2010) was used in the present study (9). The loudspeaker for the noise signal was placed behind the subjects at the same distance as the loudspeaker for speech $(80 \mathrm{~cm})$. Children were instructed to look at a soft toy on the front loudspeaker to avoid their head rotation during the speech recognition test.

\section{Speech recognition test with the FM system in noisy environments}

The FM system used in the present study consisted of a radio transmitter with a microphone (Inspiro FM transmitter and Roger digital wireless transmitter, Phonak Inc., Switzerland) and an open-fit FM receiver (iSense micro FM receiver or Roger focus receiver, Phonak Inc., Switzerland). In the audiometric testing room, the radio transmitter was placed at a distance of $20 \mathrm{~cm}$ away from the loudspeaker, facing subjects who were fitted with an FM receiver into the normal hearing ear, because in a classroom, the transmitter hangs on the teacher's neck at a distance of $20 \mathrm{~cm}$ from the mouth. Japanese monosyllables from the 57-S word list were presented at $50 \mathrm{~dB}$ time-averaged SPL from the front loudspeaker, facing a subject who sat at a distance of $80 \mathrm{~cm}$ in a quiet environment. The Japanese monosyllables were then presented in noisy environments of $0 \mathrm{~dB}$ and $-5 \mathrm{~dB}$ $\mathrm{SNR}$, in which WN was presented at 50 and $55 \mathrm{~dB}$ SPL from another loudspeaker placed behind the subject at a distance of $80 \mathrm{~cm}$ (Fig. 1B). Children were instructed to look at a soft toy on the front loudspeaker to avoid head rotation during the speech recognition test.

Speech recognition test with a cartilage conduction hearing aid in noisy environments

The cartilage conduction hearing aid used in the present study consisted of a transducer and sound processor (HB-J1CC, Rion Co., Ltd, Japan). The transducer is $11.9 \times 7.8 \times 4.7 \mathrm{~mm}$ and its weighs $0.9 \mathrm{~g}$. Because cartilage conduction does not require the transducer to exert strong and sustained pressure on the cartilage (5), it could be attached the cartilage of microtia with double-stick tape. The sound processor has nonlinear amplification, noise reduction, and feedback suppression. The adjustment was performed using PC software. The cartilage conduction hearing aid was fitted into the ear with aural atresia. Gains at 500, 1000 , and $2000 \mathrm{~Hz}$ were first adjusted to at least more than halfgain. Thereafter, fine-tuning was performed according to the audiometric test, subjective complaints, and feedback problem (6). In the audiometric testing room, children were fitted with a cartilage conduction hearing aid into the affected ear. Japanese monosyllables from the 57-S word list were presented at $50 \mathrm{~dB}$ time-averaged SPL from the front loudspeaker, facing a subject who sat at a distance of $80 \mathrm{~cm}$ in a quiet environment. The Japanese monosyllables were then presented in noisy environments of $0 \mathrm{~dB}$ and $-5 \mathrm{~dB}$ SNR, in which $\mathrm{WN}$ was presented at 50 and 55 $\mathrm{dB}$ SPL from another loudspeaker placed behind the subject at a distance of $80 \mathrm{~cm}$ (Fig. 1C). Children were instructed to look at a soft toy on the front loudspeaker to avoid head rotation during speech recognition test.

\section{Statistical analysis}

Statistical analysis was done using two-factor factorial analysis of variance and one factor analysis of variance with Scheffe's F post-hock test (Statcel version 3, OMS Publishing Inc, Saitama, Japan). $\mathrm{p}<0.05$ was considered statistically significant.
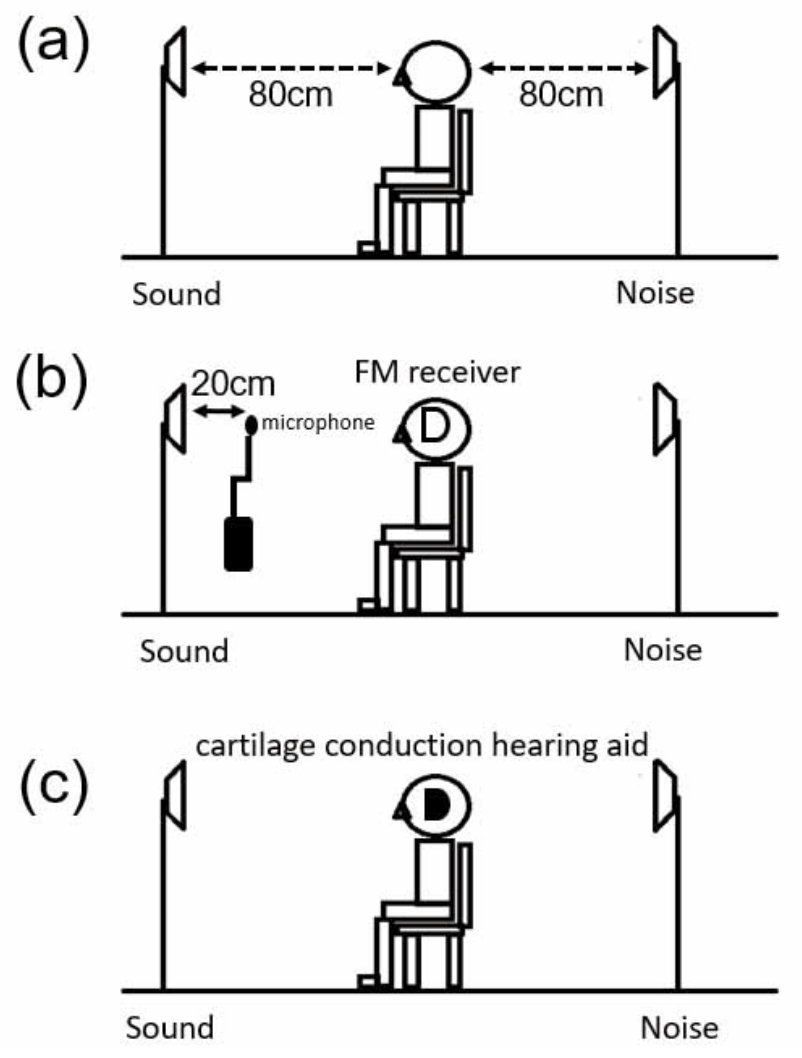

Fig 1a,b Speech recognition test in noisy environments without (a) and with (b) the FM system fitted into the normal hearing ear and (c) the cartilage conductive hearing aid fitted into the affected ear. Japanese monosyllables were presented at $50 \mathrm{~dB}$ time-average sound pressure level (SPL) from the front loudspeaker and white noise was presented at 50 or $55 \mathrm{~dB}$ SPL from the rear loudspeaker. 


\section{RESULTS}

In the children with bilateral normal hearing $(n=12)$, the speech recognition score was $95.2 \pm 3.9 \%$ in a quiet environment, and $85.5 \pm 3.8 \%$ and $70.8 \pm 6.7 \%$ in noisy environments of 50 and $55 \mathrm{~dB} \mathrm{WN}$ (0 and $-5 \mathrm{~dB}$ SNR), respectively. Thus, in bilateral normal hearing children, the speech recognition score was significantly decreased in a noisy environment of 55 $\mathrm{dB} \mathrm{WN}$, compared with those in a quiet environment. In the children with unilateral conductive hearing loss secondary to congenital aural atresia $(n=8)$, the speech recognition score was $87.5 \pm 4.2 \%$ in a quiet environment, and $62.8 \pm 10.7 \%$ and $45.1 \pm 11.9 \%$ in noisy environments of 50 and $55 \mathrm{~dB} \mathrm{WN}$, respectively. Thus, in children with unilateral congenital aural atresia, the speech recognition score was significantly decreased in noisy environments of 50 and $55 \mathrm{~dB}$ WN, compared with those in a quiet environment. In noisy environments of 50 and $55 \mathrm{~dB} W N$, the speech recognition score in children with unilateral conductive hearing loss secondary to congenital aural atresia was significantly decreased, compared those in children with bilateral normal hearing (Fig. 2).

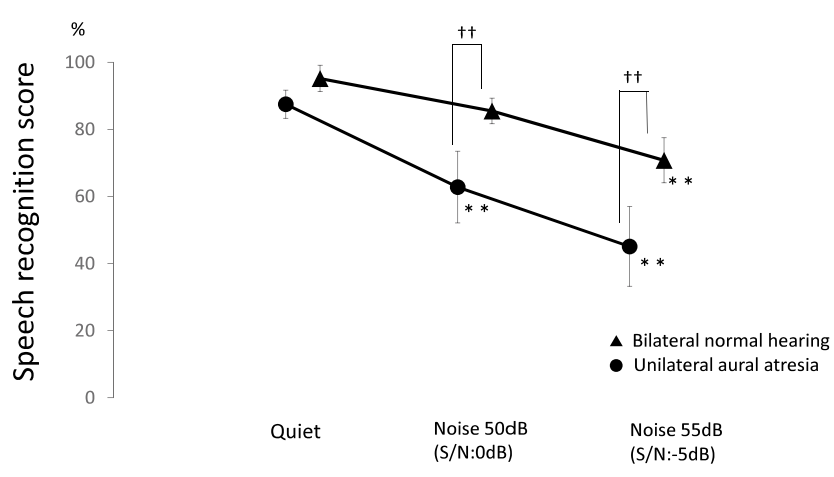

Fig 2 Effects of noise on the speech recognition ability in children with unilateral conductive hearing loss secondary to congenital aural atresia $(n=8)$ and bilateral normal hearing $(n=12)$. Circles and triangles represent children with unilateral conductive hearing loss and with bilateral normal hearing, respectively. ${ }^{* *} p<0.01$ vs. quiet, $\dagger<0.01$ between groups. Data presented as mean \pm standard deviation.

In the group of elementary school-aged children with unilateral conductive hearing loss secondary to congenital aural atresia $(n=4)$, the speech recognition score was $89.0 \pm 1.2 \%$ in a quiet environment, and $69.0 \pm 7.0 \%$ and $53.3 \pm 9.9 \%$ in noisy environments of 50 and $55 \mathrm{~dB} \mathrm{WN}$, respectively. Thus, in this group, the speech recognition score was significantly decreased in noisy environments of 50 and $55 \mathrm{~dB}$ WN, compared with those in a quiet environment. When they were aided by an FM receiver into the normal hearing ear, the speech recognition score was $91.0 \pm 3.5 \%$ in a quiet environment, and $81.0 \pm 4.8 \%$ and $71.0 \pm 6.6 \%$ in noisy environments of 50 and $55 \mathrm{~dB} \mathrm{WN}$, respectively. The speech recognition score when aided with the FM system was still significantly decreased in the noisy environment of $55 \mathrm{~dB} \mathrm{WN}$, compared with those in a quiet environment. However, the speech recognition score when aided with the FM system was significantly increased in noisy environments of 55 dB WN, compared those when unaided (Fig. 3).

Among the children tested, both school- and preschool-aged children with unilateral conductive hearing loss secondary to



Fig 3 Effects of the FM system fitted into the normal hearing ear on the speech recognition ability in noisy environments in children with unilateral conductive hearing loss secondary to congenital aural atresia. Circles and diamonds represent unaided and aided with Open-fit FM system into the normal hearing ear, respectively. $* p<0.05, * * p<0.01$ vs. quiet; $\uparrow p<0.05$ between unaided and aided with the FM system. Data presented as mean \pm standard deviation. $\mathrm{n}=4$.

congenital aural atresia $(n=5)$ were aided by a cartilage conductive hearing aid into the affected ear. The speech recognition score without a cartilage conductive hearing aid was $86.8 \pm 5.4 \%$ in a quiet environment, and $58.0 \pm 9.9 \%$ and $38.8 \pm 7.6 \%$ in noisy environments of 50 and $55 \mathrm{~dB}$ WN, respectively. Thus, the speech recognition score was significantly decreased in noisy environments of 50 and $55 \mathrm{~dB}$ WN, compared with those in a quiet environment. When they were aided by a cartilage conduction hearing aid attached into the cartilage of microtia, the speech recognition score was $88.8 \pm 7.2 \%$ in a quiet environment, and $69.2 \pm 7.0 \%$ and $43.2 \pm 12.6 \%$ in noisy environments of 50 and 55 $\mathrm{dB} W \mathrm{~W}$, respectively. The speech recognition score when aided with a cartilage conductive hearing aid was still significantly decreased in the noisy environment of $55 \mathrm{~dB}$ WN, compared with those in a quiet environment. However, the speech recognition score when aided with a cartilage conduction hearing aid tended to be increased in the noisy environment of $50 \mathrm{~dB} \mathrm{WN}(p=0.07)$, compared with those when unaided (Fig. 4).

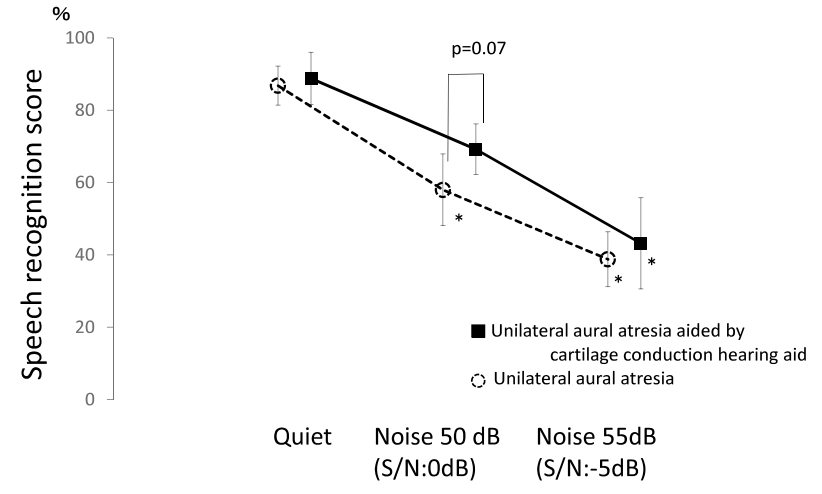

Fig 4 Effects of the cartilage conductive hearing aid fitted into the affected ear on the speech recognition ability in noisy environments in children with unilateral conductive hearing loss secondary to congenital aural atresia. Circles and squares represent unaided and aided with the cartilage conductive hearing aid attached to the cartilage of microtia, respectively. ${ }^{*} p<0.01$ vs. quiet. Data presented as mean \pm standard deviation. $\mathrm{n}=5$. 


\section{DISCUSSION}

In the present study, the speech recognition ability of Japanese children with unilateral conductive hearing loss secondary to congenital aural atresia was decreased in noisy environments of 0 and $-5 \mathrm{~dB}$ SNR, compared with that in a quiet environment. Moreover, their speech recognition ability was decreased in noisy environments of 0 and $-5 \mathrm{~dB}$ SNR, compared with that of age-matched children with bilateral normal hearing. Unilateral hearing loss is a disruption of binaural processing of the auditory system including binaural summation, head shadow effect and localization $(11,12,13)$. Our previous study reported that the acoustic condition in Japanese elementary school classrooms was a -6.9 dB SNR (14). Taken together, the present findings suggest that Japanese children with unilateral conductive hearing loss secondary to congenital aural atresia encounter difficulty understanding teachers' and classmates' speech in the classroom setting, with a resulting risk of academic failure.

Children with unilateral congenital aural atresia require some sort of resource assistance, including preferential seating, the FM system, bone-conductive hearing aids, a bone-anchored hearing aid (BAHA) or atresia surgery (4). The question of which resource will best help children with unilateral congenital aural atresia has not been answered. In the present study, an FM receiver fitted into the normal hearing ear improved the speech recognition ability of children with unilateral congenital aural atresia in the noisy environment of $-5 \mathrm{~dB}$ SNR similar to the listening condition of elementary school classrooms in Japan. Because the FM system picks up the relevant teacher's voice over a short distance and delivers it to the listener's ear through radio waves that do not interfere with surrounding ambient noises, the FM system can directly improve the SNR of auditory signals. Therefore, the present findings suggest that the FM system can be recommended to children with unilateral congenital aural atresia as a form of resource assistance for the improvement of speech recognition in noisy elementary school classrooms in Japan. It was reported that the FM system improved speech recognition in individual children with unilateral hearing loss, compared with CROS (15). We have also reported that the FM system fitted into the normal hearing ear improved the speech recognition ability of children with unilateral severe-to-profound sensorineural hearing loss in noisy environments (14).

Although use of a bone-conductive hearing aid, especially a BAHA in children with bilateral congenital aural atresia is unquestioned. But, the efficacy of a BAHA in children with unilateral congenital aural atresia remains a difficult question and has not been well studied. In adults with unilateral conductive hearing loss, the sound localization is significantly better with a BAHA than that in adults with unilateral deafness using a BAHA (16). On the other hand, it was reported that children with unilateral conductive hearing loss showed no difference in sound localization between aided and unaided conditions (17).

Recently, the cartilage conductive hearing aids have been developed and are now commercially available in Japan. Congenital aural atresia is almost always accompanied by auricular malformation (18), and all children with unilateral congenital aural atresia enrolled in the present study had a microtia. Because cartilage conduction does not require the transducer to exert strong and sustained pressure on the cartilage (5), it could be attached the cartilage of microtia with double-stick tape. The cartilage conductive hearing aid fitted into the affected ear tended to improve the speech recognition ability of children with unilateral congenital aural atresia in noisy environments of 0 $\mathrm{dB}(\mathrm{p}=0.07)$, but not $-5 \mathrm{~dB}$ SNR. The present findings showed a limited benefit of cartilage conduction hearing aid for speech recognition in noisy environments in children with unilateral congenital aural atresia, probably because they were too young (averaged age : 7.0 years) and had no previous hearing aid experience.

\section{CONCLUSION}

The present study demonstrated that the speech recognition ability of Japanese children with unilateral moderate or moderate-severe conductive hearing loss secondary to congenital aural atresia was decreased in noisy environments similar to the listening conditions in elementary school classrooms in Japan, when compared with that of age-matched children with bilateral normal hearing. An FM receiver fitted into the normal hearing ear improved the speech recognition ability in the noisy environments, suggesting that the FM system can be recommended to be used in classrooms to reduce the risk of learning difficulty and academic failure in children with unilateral congenital aural atresia. A cartilage conduction hearing aid may be a promising resource for assistance of children with unilateral congenital aural atresia.

\section{CONFLICT OF INTEREST}

The authors declare no conflicts of interest in this study.

\section{ACKNOWLEDGEMENTS}

We thank Dr. Tadashi Nishimura for his help with cartilage conduction hearing aid fitting.

\section{REFERENCES}

1. Bess $\mathrm{FH}$, Tharpe AM : Unilateral hearing impairment in children. Pediatrics $74: 206-216,1984$

2. Lieu JE : Speech-language and educational consequences of unilateral hearing loss in children. Arch Otolaryngol Head Neck Surg 130 : 524-530, 2004

3. Jensen DR, Grames LM, Lieu JE : Effects of aural atresia on speech development and learning : retrospective analysis from a multidisciplinary craniofacial clinic. JAMA Otolaryngol Head Neck Surg 139 : 797-802, 2013

4. Kesser BW, Krook K, Gray LC : Impact of unilateral conductive hearing loss due to aural atresia on academic performance in children. Laryngoscope 123 : 2270-2275, 2013

5. Hosoi H, Nishimura T, Shimokura R, Kitahara T : Cartilage conduction as the third pathway for sound transmission. Auris Nasus Larynx 46 : 151-159, 2019

6. Nishimura T, Hosoi H, Saito O, Shimokura R, Yamanaka T, Kitahara T: Cartilage Conduction Hearing Aids for Severe Conduction Hearing Loss. Otol Neurotol 39 : 65-72, 2018

7. Krishnan LA, Van Hyfte S : Management of unilateral hearing loss. Int J Pediatr Otorhinolaryngol 88:63-73, 2016

8. Nishimura $\mathrm{T}$ : Characteristics and indications of cartilage conduction hearing aids. Nihon Jibiinkoka Gakkai Kaiho $121: 1306-1307,2018$

9. Kodera K, Hosoi H, Okamoto M, Manabe T, Kanda Y, Shiraishi K, Sugiuchi T, Suzuki K, Tauchi H, Nishimura T, Matsuhira T, Ishikawa K : Guidelines for the evaluation of hearing aid fitting (2010). Auris Nasus Larynx 43 : 217-228, 2016

10. Japan Audiological Society : Speech audiometry Audiol Jpn 
$46: 622-637,2003$

11. Bess FH, Tharpe AM, Gibler AM : Auditory performance of children with unilateral sensorineural hearing loss. Ear Hear $7: 20-26,1986$

12. Bronkhorst AW, Plomp R : The effect of head-induced interaural time and level differences on speech intelligibility in noise. J Acoust Soc Am 83 : 1508-1516, 1988

13. Arsenault MD, Punch JL : Nonsense-syllable recognition in noise using monaural and binaural listening strategies. J Acoust Soc Am 105 : 1821-1830, 1999

14. Shimada A, Udaka J, Nagashima H, Chida I, Kondo E, Nakano $\mathrm{S}$, Okamoto $\mathrm{H}$, Takeda $\mathrm{N}$ : Effects of FM system fitted into normal hearing ear on speech-in-noise recognition in Japanese school-aged children with unilateral severe-to-profound hearing loss. J Med Invest 65 : 216-220, 2018

15. Updike CD : Comparison of FM auditory trainers, CROS aids, and personal amplification in unilaterally hearing impaired children. J Am Acad Audiol 5 : 204-209, 1994

16. Grantham DW, Ashmead DH, Haynes DS, Hornsby BW, Labadie RF, Ricketts TA : Horizontal plane localization in single-sided deaf adults fitted with a bone-anchored hearing aid (Baha). Ear Hear 33 : 595-603, 2012

17. Kunst SJ, Leijendeckers JM, Mylanus EA, Hol MK, Snik $\mathrm{AF}$, Cremers CW : Bone-anchored hearing aid system application for unilateral congenital conductive hearing impairment : audiometric results. Otol Neurotol $29: 2-7,2008$

18. Klockars T, Rautio J : Embryology and epidemiology of microtia. Facial Plast Surg 25 : 145-148, 2009 